\title{
QCD phase diagram and the critical point
}

\author{
Mikhail Stephanov \\ Department of Physics, University of Illinois, Chicago, IL 60607-7059 \\ and \\ RIKEN-BNL Research Center, Brookhaven National Laboratory, Upton, NY 11973
}

The recent progress in understanding the QCD phase diagram and the physics of the QCD critical point is reviewed.

\section{$\S 1$. Preamble}

Quantum Chromodynamics is one of the most remarkable theories of Nature. Its mathematical foundations are concise, yet the phenomenology which the theory describes is broad and diverse. QCD phenomenology at finite temperature and baryon number density is one of the least explored regimes of the theory. There are several experimental windows into this regime. One is the physics associated with the interior of neutron stars. Another, which is the subject of the ongoing and planned experimental programs, is the physics of heavy ion collisions.

This report is a review of recent developments in our understanding, mostly theoretical, but also experimental, of the phase diagram of QCD. There exist a number of excellent recent reviews ${ }^{1-6)}$ which discuss many of the questions addressed here as well as the related material not covered in this report. The report focuses on the physics of the critical point of QCD and its search. There are many open questions in this field, and some of the theoretical as well as experimental results and expectations discussed here might not hold under further scrutiny. Nevertheless it is hoped that this report will provide a useful contemporary guide to both theorists and experimentalists entering the field as well as a stimulating reading to the field's experts.

\section{$\S 2$. What is the QCD critical point?}

\subsection{The phase diagram}

Fig. 1 shows a sketch of the QCD phase diagram as it is perceived by a modern theorist. By a phase diagram we shall mean the information about the location of the phase boundaries (phase transitions) as well as the physics of the phases that these transitions delineate. The phase transitions are the thermodynamic singularities of the system. The system under consideration is a region (in theory, infinite) occupied by strongly interacting matter, described by QCD, in thermal and chemical equilibrium, characterized by the given values of temperature $T$ and baryo-chemical potential $\mu_{B}$. In practice, it can be a region in the interior of a neutron star, or inside the hot and dense fireball created by a heavy ion collision.

typeset using $\mathcal{P} \mathcal{T} \mathcal{P}$ TEX.cls $\langle$ Ver.0.89 $\rangle$ 


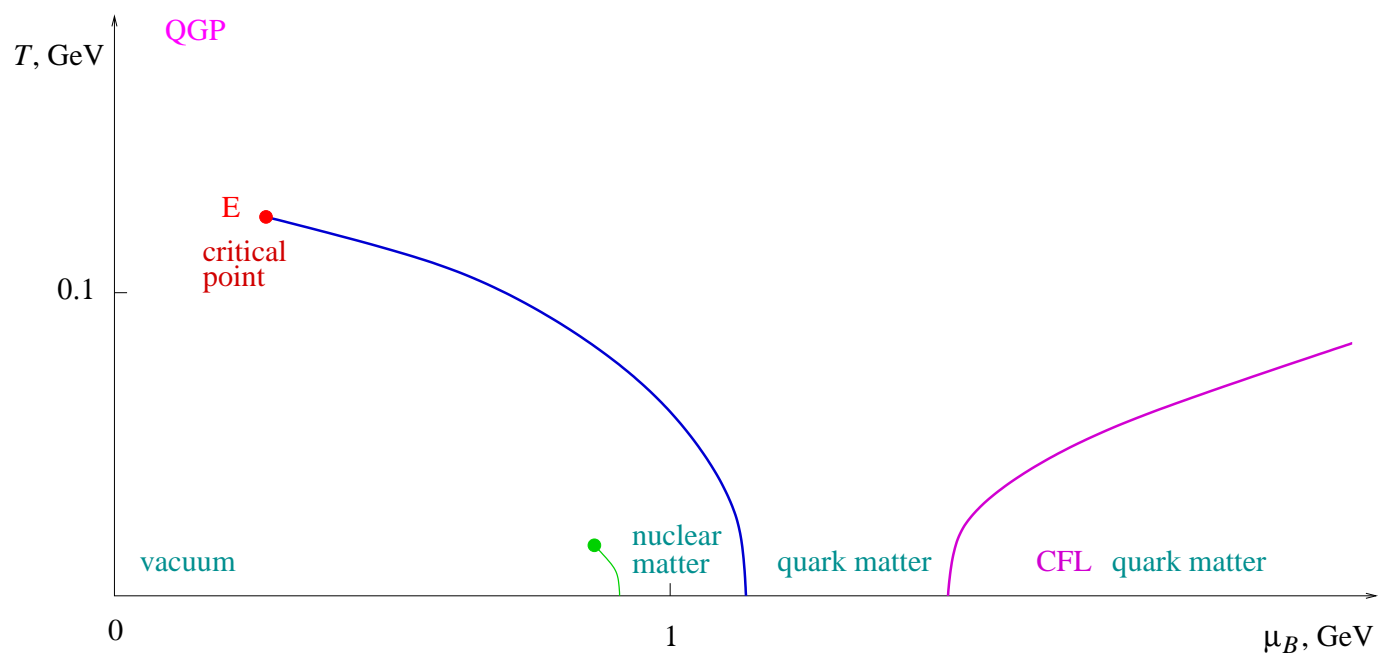

Fig. 1. QCD phase diagram

On the phase diagram, the regime of small $T$ and large $\mu_{B}$ is of relevance to neutron star physics. Because of low temperature, a very rich spectrum of possibilities of ordering can be envisaged. The line separating the Color-Flavor-Locked (CFL) phase, predicted in Ref. 7), from the higher temperature disordered phase (quarkgluon plasma, or QGP) is the most simplified representation of the possible phase structure in this region. This regime is also of particular theoretical interest because analytical controllable calculations are possible, due to asymptotic freedom of QCD. The reader is referred to the reviews ${ }^{1)-5}$ ) which cover the recent developments in the study of this domain of the phase diagram.

The region of the phase diagram more readily probed by the heavy ion collision experiments is that of rather large $T \sim 100 \mathrm{MeV}$, commensurate with the inherent dynamical scale in QCD, and small to medium chemical potential $\mu_{B} \sim 0-600$ $\mathrm{MeV}$. Theorists expect that this region has an interesting feature - the end point of the first order phase transition line, the critical point marked $E$ on Fig. 1. The physics of this point is the focus of the review.

\subsection{Why should there be a critical point?}

The argument (which is not a proof) that the point $E$ must exist is short, and is based on a small number of reasonable assumptions. The two basic facts that it relies on are as follows:

(1) The temperature driven transition at zero $\mu_{B}$ is not a thermodynamic singularity. Rather, it is a rapid, but smooth, crossover from the regime describable as a gas of hadrons, to the one dominated by internal degrees of freedom of QCD quarks and gluons. This is the result of finite $T$ lattice calculations. ${ }^{8)}$

(2) The $\mu_{B}$ driven transition at zero $T$ is a first order phase transition. This conclusion is less robust, since the first principle lattice calculations are not controllable in this regime (naive Euclidean formulation of the theory suffers from the notorious sign problem at any finite $\mu_{B}$ ). Nevertheless a number of different model 
approaches ${ }^{9)-16)}$ (see Section 3.3) indicate that the transition in this region is strongly first order.

(3) The last step of the argument is a logical product of (1) and (2). Since the first order line originating at zero $T$ cannot end at the vertical axis $\mu_{B}=0$ (by virtue of (1)), the line must end somewhere in the midst of the phase diagram.

The end point of a first order line is a critical point of the second order. This is by far the most common critical phenomenon in condensed matter physics. Most liquids possess such a singularity, including water. The line which we know as the water boiling transition ends at pressure $p=218$ atm and $T=374^{\circ} \mathrm{C}$. Along this line the two coexisting phases (water and vapor) become less and less distinct as one approaches the end point (the density of water decreases and of vapor increases), resulting in a single phase at this point and beyond.

In QCD the two coexisting phases are hadron gas (lower $T$ ), and quark-gluon plasma (higher $T$ ). What distinguishes the two phases? As in the case of water and vapor, the distinction is only quantitative, and more obviously so as we approach the critical point. Rigorously, there is no good order parameter which could distinguish the two phases qualitatively. The chiral condensate, $\langle\bar{\psi} \psi\rangle$, which comes closest to being an order parameter, is non-zero in both phases because of the finite bare quark mass. Deconfinement, although a useful concept to discuss the transition from hadron to quark-gluon plasma, strictly speaking, does not provide a good order parameter. Even in vacuum $(T=0)$ the confining potential cannot rise infinitely - a quark-antiquark pair inserted into the color flux tube breaks it. The energy required to separate two test color charges from each other is finite if there are light quarks.

\subsection{Critical or tricritical?}

There is an idealization of QCD where the distinction between the hadron gas and quark gluon plasma is sharp. It describes the world with massless quarks $m_{q}=0$. In this limit of QCD with 2 massless quarks (up and down) the chiral symmetry $\mathrm{SU}(2)_{V} \times \mathrm{SU}(2)_{A}$ is exact. Although interactions respect this symmetry, it is spontaneously broken in the QCD vacuum to $\mathrm{SU}(2)_{V}$, and the Goldstone theorem demands 3 massless bosons - the pions. This breaking is a result of nonperturbative dynamics in QCD (instantons provide a natural mechanism).

The breaking of the global symmetry, such as the chiral symmetry, can be thought of as establishment of the long-range order in the vacuum. It is the order which dictates the preferred $\mathrm{SU}(2)_{A}$ direction for all points in space, and over which the pions are quantized "ripples". At sufficiently high $T$, the order is melted as in any other such system (compare, e.g., to the disordering of the ferromagnet at Curie temperature). The chiral symmetry is restored. The two phases must be separated by a thermodynamic singularity - a phase transition.

This argument can be made more rigorous by considering the order parameter, $\langle\bar{\psi} \psi\rangle$, the expectation value, or the condensate, of a field transforming non-trivially under the broken symmetry. Consider a fixed value of $\mu_{B}$, e.g., $\mu_{B}=0$. The chiral condensate $\langle\bar{\psi} \psi\rangle$ as a function of $T$ is identically zero (by symmetry) for all temperatures above some value $T_{c}$ and is nonzero function of $T$ below (symmetry breaking). Such a function cannot be analytic. A singularity must occur at $T_{c}$. 
In QCD, lattice calculations show that this singularity is a second order phase transition if $T_{c}$ is approached at $\left.\left.\mu_{B}=0 .{ }^{8)}, 17\right)-23\right)$ At other values of $\mu_{B}$ the critical temperature $T_{c}$ is different, but the line of transitions $T_{c}\left(\mu_{B}\right)$ cannot terminate, since any path from the vacuum $T=\mu_{B}=0$ to the high $T$ phase must cross a singularity. Somewhere in the midst of the phase diagram the order of the transition should change to first order (according to point (2) of Section 2.2).*) The point where this happens is the tricritical point. The resulting phase diagram is illustrated in Fig. 2.

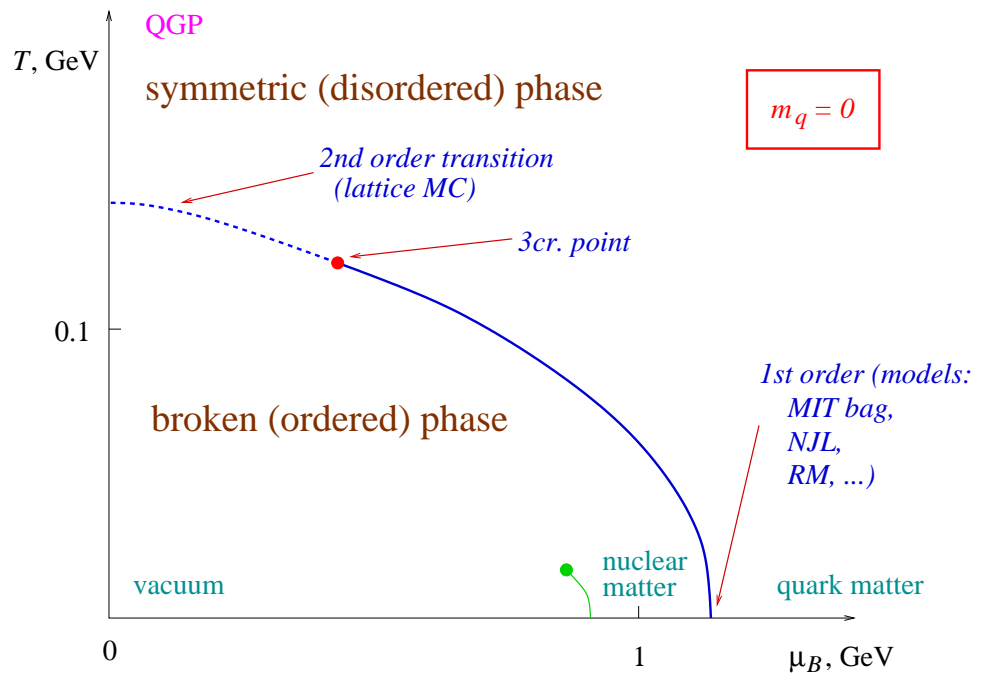

Fig. 2. Phase diagram of QCD with two massless quarks. The chiral symmetry order parameter qualitatively distinguishes two phases: $\langle\bar{\psi} \psi\rangle \neq 0$ in the broken phase and $\langle\bar{\psi} \psi\rangle=0$ in the symmetric phase.

Once the quark mass $m_{q}$ is turned back on, the distinction between the symmetric and broken phases is blurred, and the second order phase transition is replaced by a smooth crossover. The situation is analogous to the ferromagnet - an arbitrary small magnetic field (the analog of $m_{q}$ ) smooths away the Curie singularity (Fig. 3). The first order phase transition, on the other hand, is associated with a finite discontinuity of the order parameter and cannot be removed by an arbitrarily small perturbation $m_{q} \neq 0$. Thus we arrive back at

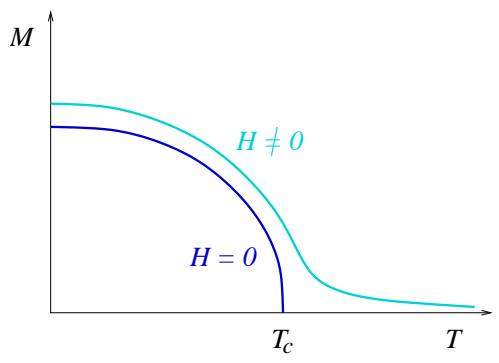

Fig. 3. The order parameter vs temperature in a Curie ferromagnet with zero and non-zero applied magnetic field. In QCD, the chiral order parameter $\langle\bar{\psi} \psi\rangle$ behaves similarly as a function of $T$ at $m_{q}=0$ and $m_{q} \neq 0$. the diagram in Fig. 1.

It is also useful to take a look at Fig. 4, where the 2-dimensional $T \mu_{B}$ phase

*) We also assume that there is only one transition between the broken and symmetric phases. 


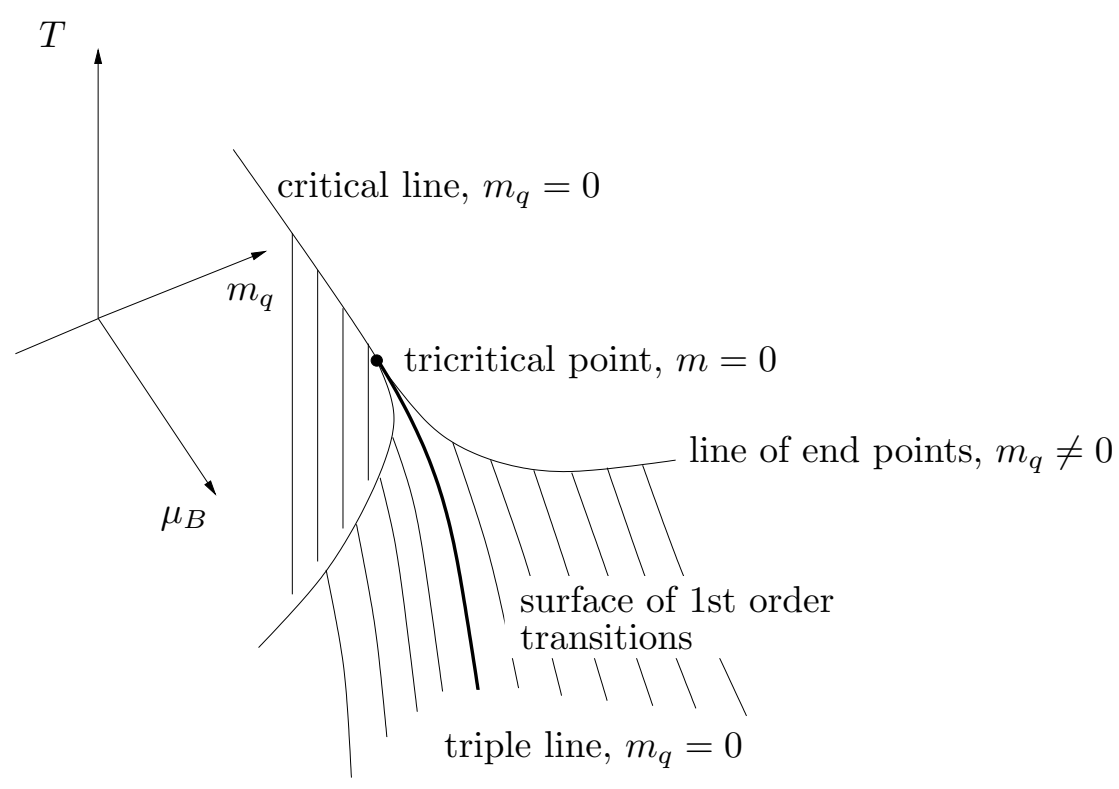

Fig. 4. A three-dimensional view $\left(T, \mu_{B}, m_{q}\right)$ of the QCD phase diagram near the tricritical point.

diagram is extended to 3 -dimensions by adding the quark mass $m_{q}$ as the third axis. One can see that the second order transition line at $m_{q}=0$ does not extend into $m_{q} \neq 0$. This line can be seen as a boundary of the coexistence surface of the two spontaneously broken phases with $\langle\bar{\psi} \psi\rangle$ of opposite signs. A first order line ending at a critical point, on the other hand, exists for all nonzero (small) $m_{q}$, thus making up a surface which looks like two wings in Fig. 4. The tricritical point can be seen as the end of a first order line where 3 phases coexist (line of triple points).

Another useful sketch is made in Fig 5. It shows, in a schematic way, the shape of the effective potential in various regions around the tricritical point. One can see that the three minima, which are equally deep on the triple line fuse into one minimum at the tricritical point.

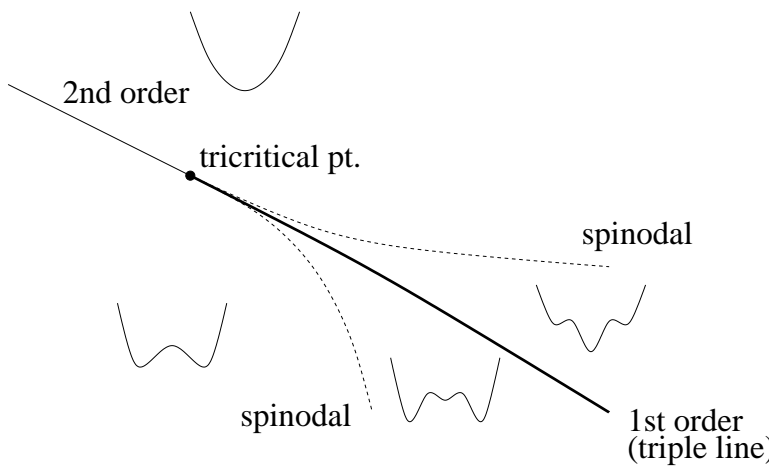

Fig. 5. Illustration of the shape of the effective potential for the chiral order parameter near the tricritical point in the $m_{q}=0$ plane. Two additional (spinodal) lines, not present in Fig. 4 indicate the boundary of the existence of metastable minima. 


\subsection{Critical behavior: static and dynamic universality class}

Determining properties of QCD (equation of state, correlation functions, etc.) near the critical point is difficult, for the same reason as it is difficult to find the location of the critical point (see next Section). However, as it is the case for any critical point, singular properties, such as critical exponents, can be determined using universality arguments.

According to the scaling postulate, central to the theory of critical phenomena, ${ }^{24)}$ all singular contributions to the thermodynamic quantities are powers of the correlation length $\xi$, which diverges at the critical point. These powers, or critical exponents, are universal, in the sense that they depend only on the degrees of freedom in the theory and their symmetry, but not on the other details of the interactions. Very different physical systems may belong to the same universality class, as far as their critical behavior is concerned.

One should distinguish static and dynamic universality classifications. ${ }^{25)}$ From the point of view of static critical phenomena, the QCD critical point falls into the universality class of the Ising model. This is a consequence of the fact that at $m_{q} \neq 0$ no symmetry remains which would require the order parameter to have more than just one component. The field theory which describes the static critical behavior, the one-component $\phi^{4}$ theory in 3 dimensions, has the critical exponents of the Ising model. *)

What is the nature of this order parameter? It can be taken as the value of the chiral order parameter $\bar{\psi} \psi$ (often called $\sigma$ ), since it is distinct in two phases coexisting across the first order phase transition terminating in the critical point. As a result, the static (equal-time) correlation function $\langle\bar{\psi} \psi(\boldsymbol{x}) \bar{\psi} \psi(\boldsymbol{y})\rangle$ develops divergent correlation length:

$$
\langle\bar{\psi} \psi(\boldsymbol{x}) \bar{\psi} \psi(\mathbf{0})\rangle_{\mathrm{c}} \sim \begin{cases}\frac{1}{|\boldsymbol{x}|^{1+\eta}}, & |\boldsymbol{x}| \ll \xi ; \\ e^{-|\boldsymbol{x}| / \xi}, & |\boldsymbol{x}| \gg \xi ;\end{cases}
$$

where $\langle\bar{\psi} \psi(\boldsymbol{x}) \bar{\psi} \psi(\mathbf{0})\rangle_{\mathrm{c}} \equiv\langle\bar{\psi} \psi(\boldsymbol{x}) \bar{\psi} \psi(\mathbf{0})\rangle-\langle\bar{\psi} \psi\rangle^{2}$. The correlation length diverges, $\xi \rightarrow \infty$, as we approach the critical point. For the Ising universality class $\eta \approx 0.04$.

Another interesting quantity, both from theoretical and experimental points of view, is the baryon number density $n_{B}(\boldsymbol{x})$. Because symmetry (or, rather, the absence of such) allows mixing of $n_{B}(\boldsymbol{x})$ with $\bar{\psi} \psi(\boldsymbol{x})$, the divergence of the baryon number susceptibility is related to the divergence of the correlation length $\xi$ :

$$
\frac{\partial n_{B}}{\partial \mu_{B}}=\int d^{3} \boldsymbol{x}\left\langle n_{B}(\boldsymbol{x}) n_{B}(\mathbf{0})\right\rangle_{\mathrm{c}} \sim \int d^{3} \boldsymbol{x}\langle\bar{\psi} \psi(\boldsymbol{x}) \bar{\psi} \psi(\mathbf{0})\rangle_{\mathrm{c}} \sim \xi^{2-\eta} .
$$

The baryon number density also jumps across the first order phase transition. One can equally well use $n_{B}$ as the degree of freedom in the effective theory near

*) As another example, consider any of the critical points on the 2 nd order line at $m_{q}=0$ on Fig. 2. Because of the $\mathrm{O}(4) \sim \mathrm{SU}(2)_{V} \times \mathrm{SU}(2)_{A}$ symmetry, which is restored at this critical point, the order parameter must carry 4 components - sigma and 3 pions $(\sigma, \pi)$. The resulting field theory describes the universality class of the $O(4)$ ferromagnet. ${ }^{26)-28)}$ 
the critical point, or any linear combination of $\bar{\psi} \psi$ and $n_{B}$ (or any other field which can mix with $\bar{\psi} \psi$ ) which is discontinuous across the first order phase transition. Regardless of the choice, there is only one order parameter, as far as the static critical behavior is concerned.

The situation is similar but a little more complicated if one considers dynamic critical behavior, e.g., the singularities of kinetic coefficients, etc. The scaling postulate is similar in this case, but the universality classes are now determined by the degrees of freedom which define the effective hydrodynamic theory near the critical point. ${ }^{25)}$ In this case the fundamental difference between $\bar{\psi} \psi$ and $n_{B}$ fields is that the latter is a conserved density. The hydrodynamic equations for $n_{B}$ are diffusive, while the dynamics of $\bar{\psi} \psi$ is relaxational. Because the two modes mix, there is, again, only one independent hydrodynamic variable, and it is diffusive. ${ }^{29), 30)}$ This mode involves fluctuations of both $\bar{\psi} \psi$ and $n_{B}$ in a fixed proportion. The fluctuations of $\bar{\psi} \psi$ alone relax on a finite time scale even at the critical point.*)

The complete hydrodynamic theory near the critical point must also involve the energy and momentum densities. Once the hydrodynamic equations are written down, and the mixing between $\bar{\psi} \psi, n_{B}$ and the energy density is taken into account, one finds the theory equivalent to the one describing the liquid-gas phase transition, model $\mathrm{H}$ in the classification of Ref. 25). One consequence of this theory, interesting from phenomenological point of view, is the vanishing of the baryon number diffusion rate at the critical point: $D \sim \xi^{-x_{D}}$, with exponent $x_{D} \approx 1$. $^{30}$ )

\section{$\S 3$. Where is the critical point? Theory}

Theoretically, finding the coordinates $\left(T, \mu_{B}\right)$ of the critical point is a welldefined task. We need to calculate the partition function of QCD and find the singularity corresponding to the end of the first order transition line. The Lagrangian of QCD is known, and the partition function is given by a path integral of the exponent of the QCD action, after Wick rotation to the Euclidean space (with imaginary time compactified on a torus of circumference $1 / T$ ).

Of course, calculating such an infinitely dimensional integral analytically is beyond our present abilities (perturbation theory is not an option here, in the relevant region of $T$ and $\mu_{B}$ ). We are thus left with numerical methods, i.e., lattice Monte Carlo simulation. At zero $\mu_{B}$ this method allows us to determine the equation of state of QCD as a function of $T$ and reach the conclusion (1) in Section 2.2. However, at finite $\mu_{B}$ the Nature guards its secrets better.

\subsection{Importance sampling and the sign problem}

The notorious sign problem has been known to lattice experts since early days of this field. Calculating the partition function using Monte Carlo method hinges on the fact that the exponent of the Euclidean action $S_{E}$ is a positive definite function of its variables (values of the fields on the lattice). This allows one to limit calculation

*) A related observation, that the sigma pole mass does not vanish at the critical point in the large- $N$ NJL model, was made in Ref. 14) and confirmed in Ref. 29). 
to a relatively small set of field configurations randomly picked with probability proportional to the value of $\exp \left(-S_{E}\right)$. The number of such configurations needed to achieve reasonable accuracy is vastly smaller than the total number of possible configurations. The latter is exponentially large in the size $V$ of the system, or, the number of the degrees of freedom: $\exp ($ const $\cdot V)$. The method, also known as importance sampling, utilizes the fact that the vast majority of these configurations contribute a tiny fraction because of the exponential suppression by $\exp \left(-S_{E}\right)$. Only configurations with sizable $\exp \left(-S_{E}\right)$ are important.

At $\mu \neq 0$ the action $S_{E}$ is complex. What configurations are important then? A number of ways to circumvent the problem have been tried. For example, using the modulus of $\exp \left(-S_{E}\right)$ as a measure of importance, or the value of $\exp \left(-S_{E}\right)$ at zero $\mu_{B}$. Unfortunately, none work, at present.

\subsection{The overlap problem}

For the latter choice, $\left.\exp \left(-S_{E}\right)\right|_{\mu_{B}=0}$, the problem can be understood physically and is known as the overlap problem. The important configurations at $\mu_{B}=0$ are different than those of $\mu_{B} \neq 0$. How bad is this quantitatively? At finite volume, even at $\mu_{B}=0$, the configurations important for $\mu_{B} \neq 0$ pop up, but with a small probability. This probability is exponentially small as volume $V \rightarrow \infty$ : $\exp (-$ const $\cdot V)$. When we calculate the partition function using this method, we correct for this by multiplying the contribution of these rare configurations by the factor $\exp \left(+\left.S_{E}\right|_{\mu_{B}=0}-S_{E}\right)$. The procedure is termed reweighting. $\left.{ }^{*}\right)$ The reweighting factor is exponentially large as $V \rightarrow \infty$ - both the magnitude and the complex phase are $\exp ($ const $\cdot V)$. Fluctuations, or statistical noise, in the exponentially tiny number of the rare important configurations completely washes out the significance of the result.

In layman's terms, imagine that we want to study ice, but can only run experiments at normal room temperature and pressure. Using the reweighting method is analogous to trying to glimpse the information by waiting for rare configurations when all the water molecules accidentally gather in one corner of the lab, forming a chunk of ice. The amount of time that this experiment would require is exponentially large as $V \rightarrow \infty$.

\subsection{Theoretical predictions}

The first lattice prediction for the location of the critical point has been reported in Ref. 32). The assumption is that, although the problem becomes exponentially difficult as $V \rightarrow \infty$, in practice, once can get a sensible approximation at finite $V$. In addition, simulations at finite $T$ might suffer lesser overlap problem because of large thermal fluctuations. ${ }^{33)}$ One can hope that if the critical point is at a small value of $\mu_{B}$, the volume $V$ may not need to be too large to achieve a reasonable accuracy. In particular, numerical estimates show ${ }^{34}$ ) that the maximal value of $\mu_{B}$ which one can reach within the same accuracy shrinks only as a power of $1 / V$.

However, it is not possible to determine this accuracy, since the exact result is

*) The reweighting method in application to finite $\mu_{B}$ calculations is known as the "Glasgow method" (reviewed in Ref. 31)). 
unknown. Normally, one would estimate the error by going to increasingly large volumes $V$, but, as discussed above, the method becomes prohibitive too quickly (exponentially) in this limit. Ultimately, the result of Ref. 32) might turn out to be a good approximation to the exact answer, but we can only tell once we have an independent result to compare it to. A qualitatively new approach is needed to overcome the QCD sign problem. *)

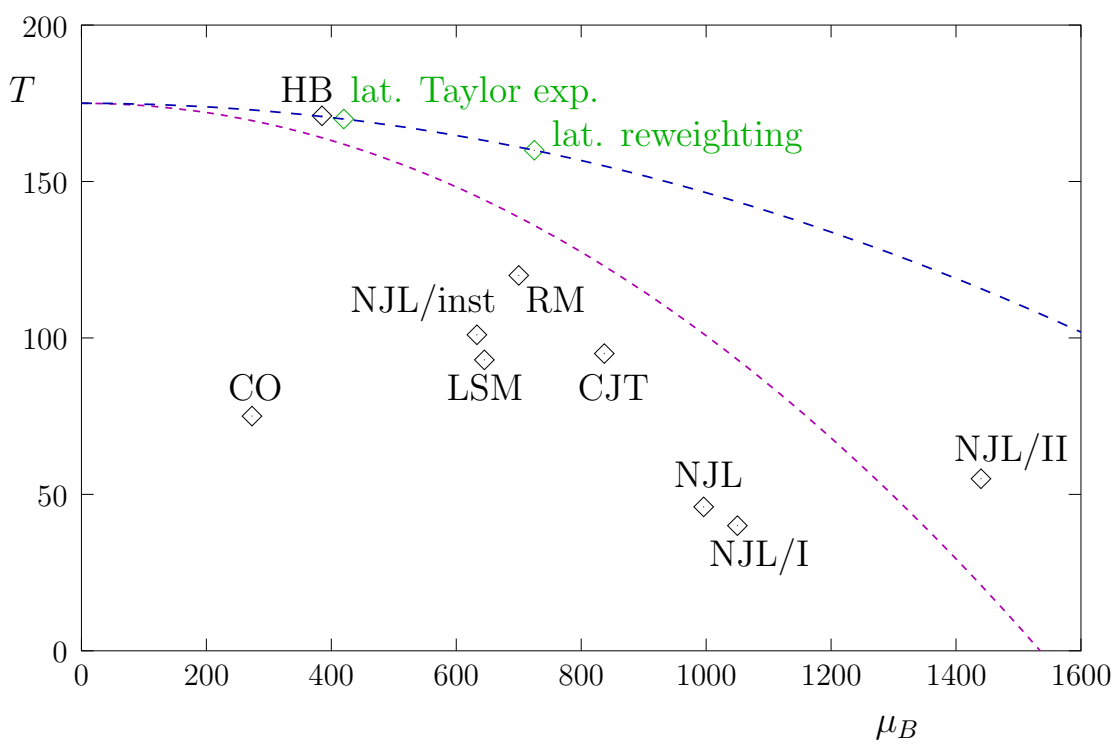

Fig. 6. Theoretical (models and lattice) predictions for the location of the critical point. The labels correspond to Table I. The two dashed lines indicate the magnitude of the slope $d^{2} T / d \mu^{2}$ obtained by lattice Taylor expansion. ${ }^{38)}$ The upper curve agrees with Ref. 39). The lower curve corresponds to smaller quark mass. Errors/uncertainties are not shown.

In the absence of a controllable (i.e., systematically improvable) method, one turns to model calculations. Many such calculations have been done. ${ }^{9)-16)}$ Figure 6 and Table I summarize the results. One can see that the predictions vary wildly. An interesting point to keep in mind is that each of these models is tuned to reproduce vacuum, $T=\mu_{B}=0$, phenomenology. Nevertheless, extrapolation to nonzero $\mu_{B}$ is not constrained significantly by this. In a loose sense, the existing lattice methods can be also viewed as extrapolations from $\mu_{B}=0$, but finite $T$.

Two new lattice approaches are being developed. Each of them has the capacity to determine the location of the critical point. One approach is based on simulations at finite imaginary values of $\mu_{B}{ }^{39)}$ and the other on Taylor expansions around $\mu_{B}=0 .{ }^{38)}$ The curvature of the phase transition line found using these methods is indicated by the upper parabola in Fig.6. Recent result ${ }^{38)}$ (lower parabola in Fig.6) seems to indicate large sensitivity of this curvature to the quark mass. This may or may not be related to the strong sensitivity of the position of the critical point to the value of the strange quark mass observed in Ref. 39). Qualitatively, one should

*) In theories similar, or approximating, the finite density QCD, the sign and/or overlap problems have been tackled recently, using various new methods see, e.g., Refs. 35)-37). 
expect that the critical point moves toward smaller $\mu_{B}$ as the strange quark mass $m_{s}$ is decreased, since for sufficiently small $m_{s}$ the chiral transition must turn first order according to renormalization group arguments. ${ }^{26)}$

\begin{tabular}{|l|r|l|l|}
\hline Source & $\left(T, \mu_{B}\right), \mathrm{MeV}$ & Comments & Label \\
\hline \hline MIT Bag/QGP & none & only 1st order, no chiral symmetry & - \\
\hline Asakawa,Yazaki '89 & $(40,1050)$ & NJL, CASE I & NJL/I \\
\hline “" & $(55,1440)$ & NJL, CASE II & NJL/II \\
\hline Barducci, et al '89-94 & $(75,273)_{\mathrm{TCP}}$ & composite operator & CO \\
\hline Berges, Rajagopal '98 & $(101,633)_{\mathrm{TCP}}$ & instanton NJL & NJL/inst \\
\hline Halasz, et al '98 & $(120,700)_{\mathrm{TCP}}$ & random matrix & RM \\
\hline Scavenius, et al '01 & $(93,645)$ & linear $\sigma$-model & LSM \\
\hline “ & $(46,996)$ & NJL & NJL \\
\hline Fodor, Katz '01 & $(160,725)$ & lattice reweighting & \\
\hline Hatta, Ikeda, '02 & $(95,837)$ & effective potential (CJT) & CJT \\
\hline Antoniou, Kapoyannis '02 & $(171,385)$ & hadronic bootstrap & HB \\
\hline Ejiri, et al '03 & $(?, 420)$ & lattice Taylor expansion & \\
\hline
\end{tabular}

Table I. Theoretical predictions of the location of the critical point. The predictions for tricritical point are indicated as 'TCP'. The last column gives the corresponding label on Fig. 6 .

\section{$\S 4$. Scanning QCD phase diagram}

Even though the exact location of the critical point is not known to us yet, the available theoretical estimates strongly indicate that the point is within the region of the phase diagram probed by the heavy-ion collision experiments. This raises the possibility to discover this point in such experiments. ${ }^{40)}$

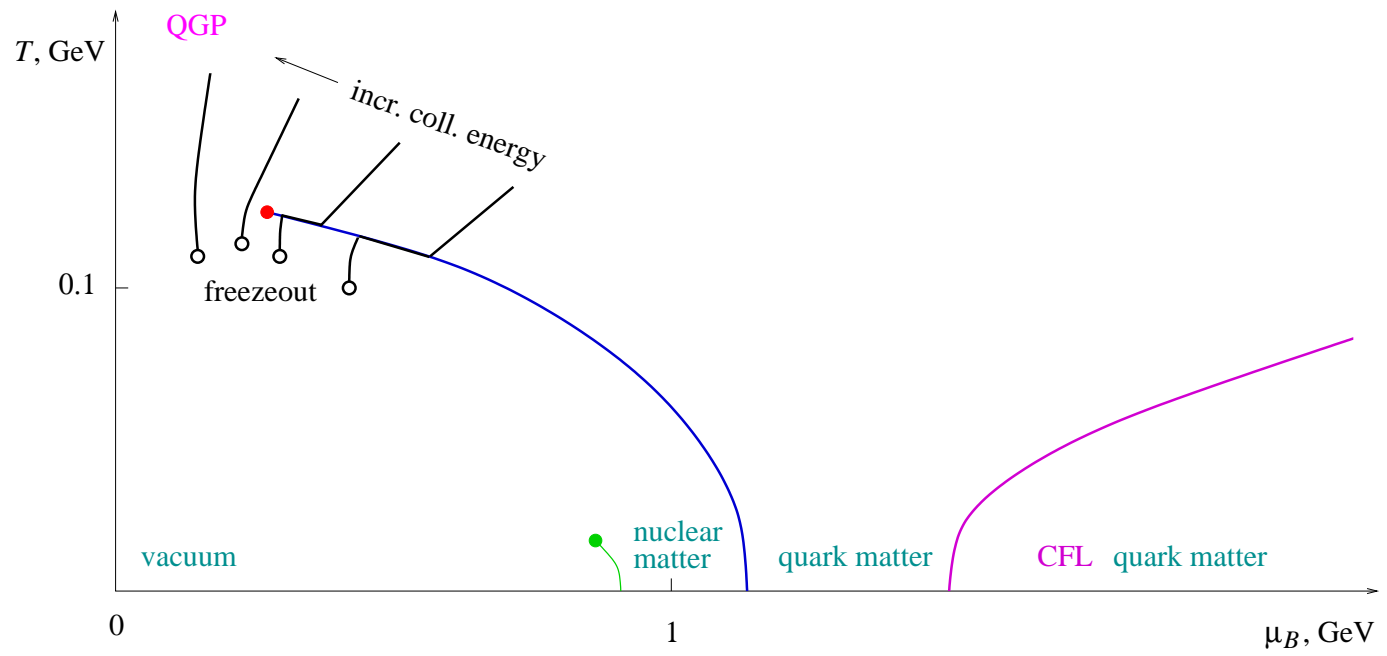

Fig. 7. Example trajectories traced by a fireball created in a heavy ion collision on the phase diagram of Fig.1. Increasing the collision energy one moves the freezeout point (empty circle) to smaller $\mu_{B}$, approaching and then receding away from the critical point.

The idea is illustrated in Fig. 7. It is known empirically that with increasing 
collision energy, $\sqrt{s}$, the resulting fireballs tend to freezeout at decreasing values of the chemical potential. This is easy to understand, since the amount of generated entropy (heat) grows with $\sqrt{s}$ while the net baryon number is limited by that number in the initial nuclei.

The trajectories on Fig. 7 terminating in the freezeout points indicate (theoretically perceived) time history of a small, but thermodynamically macroscopic, volume of the expanding fireball, at various initial collision energies. In the approximation of ideal hydrodynamics these trajectories follow lines of constant baryon per entropy ratio (baryon asymmetry), due to conservation of the baryon number and the entropy. The characteristic discontinuity of the trajectory at the first order phase transition is a result of the discontinuity of the baryon asymmetry across this transition. Because the shift is toward the end point $E$, this leads to the phenomenon of focusing: ${ }^{40)}$ the freezeout points tend to cluster near the critical point for a wide range of initial trajectory points. Therefore, wider range of $\sqrt{s}$ leads to freezeout in the critical region, making the task of finding the point somewhat easier.
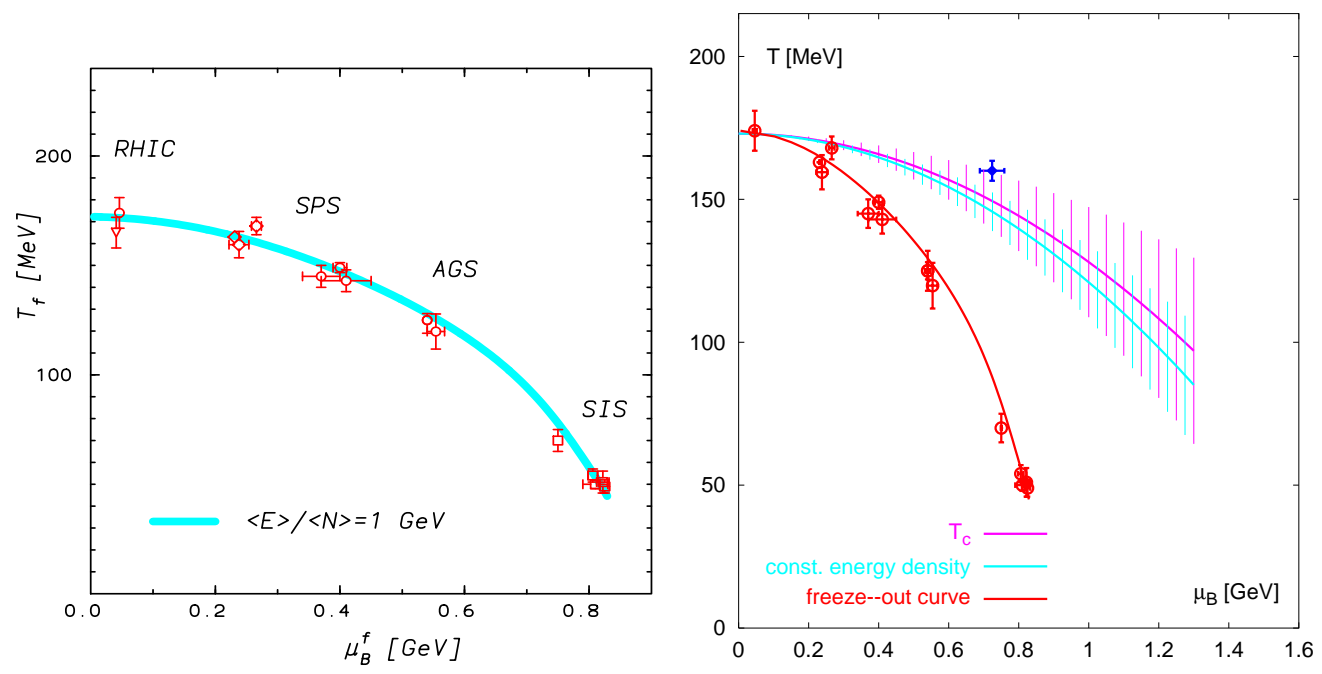

Fig. 8. The freezeout points for different heavy-ion collision experiments. On the right, the lattice results $^{32), 38), 39)}$ are superimposed. Figures are reproduced from Ref. 41).

The information about the location of the freezeout point for given experimental conditions is obtained by measuring the ratios of particle yields (e.g., baryons or antibaryons to pions), and fitting to a statistical model with $T$ and $\mu_{B}$ as parameters. Such fits are amazingly good, ${ }^{41)}$ and the resulting points for different experiments are shown in Fig. 8.

For comparison, the location of the lattice reweighting calculation result ${ }^{32}$ is superimposed on the experimental freezeout curve in Figure 8(right). It may appear that the critical point is somewhat away (higher $T$ ) from the freezeout curve. However, as emphasized already in Section 3.3, the systematic error of the lattice result is not known, since the volume $V \rightarrow \infty$ limit is unattainable using the reweighting method. Even if one takes the lattice result at face value, one still has to take into 
account the fact that the position of the critical point must shift to smaller values of $\mu_{B}$ once the quark masses (notably, the strange quark mass) are reduced toward their true values, from those used in Ref. 32). More recent lattice results ${ }^{38), 39)}$ support this expectation. The results of the model calculations (see Figure 6 and Table I) are closer to the freezeout curve than the band in Fig. 8.

Additional effect, which plays a significant role, is the critical slowing down near the point $\left.\left.E{ }^{30)}, 42\right), 43\right)$ This phenomenon is the major limiting factor (size limitation is less stringent ${ }^{42)}$ ) for the maximal correlation length that can be achieved realistically in a heavy ion collision experiment. Although, as a result, the sharpness of the signatures of the critical point is reduced, another consequence is the shift of the position (due to delay) of the maximum of the correlation length toward lower temperatures. ${ }^{43)}$

On the experimental side, one has some control over the freezeout temperature by adjusting the size of the ions. Smaller systems freeze out somewhat earlier (higher $T$ ). Also, due to the singularity in the specific heat, the freezeout occurs at higher $T$ for trajectories passing near the critical point. ${ }^{40)}$

Another interesting observation, potentially important for the search of the critical point is that of the shape of the critical region. ${ }^{16)}$ One can see in Figure 9 a model calculation of a divergent susceptibility near the critical

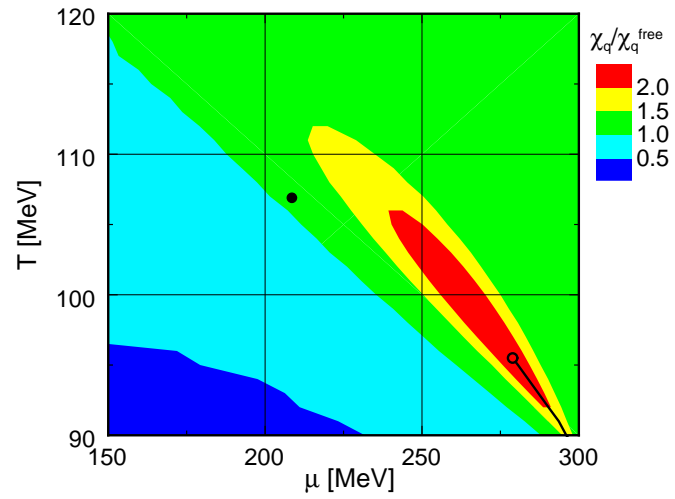

Fig. 9. The model calculation ${ }^{16)}$ of the shape of the critical region. Note that $\mu=\mu_{B} / 3$. point, which shows that the critical region is stretched in the direction of the crossover line. This shape is easy to understand remembering that, in the $\left(T, \mu_{B}, m_{q}\right)$ space (see Fig. 4$)$, the critical point is connected to the tricritical point (the black dot in Fig. 9) by a whole line of critical points.

\section{$\S 5$. Signatures: event-by-event fluctuations}

One of the actively pursued signatures of the critical point is the non-monotonous dependence on $\sqrt{s}$ (and thus, on $\mu_{B}$ ) of the event-by-event fluctuation observables. $\left.{ }^{40)}, 42\right)$ The idea can be understood qualitatively by noting that: (1) the susceptibilities diverge at the critical point, and (2) the magnitude of the fluctuations are proportional to the corresponding susceptibilities. For example, for the fluctuations of energy or charge, the well-known relations are

$$
\frac{\partial E}{\partial T}=\frac{1}{T^{2}}\left\langle(\Delta E)^{2}\right\rangle ; \quad \frac{\partial Q}{\partial \phi}=\frac{1}{T}\left\langle(\Delta Q)^{2}\right\rangle .
$$

Ideally, one could determine susceptibilities on the left-hand sides by measuring the fluctuations on the right-hand side. ${ }^{44)}$ However, practically, the measurement of 
the corresponding fluctuations, $\Delta E$ or $\Delta Q$, is not feasible because not all the particles end up in the detector. ${ }^{42), 45)}$ A more differential measure of the fluctuations needs to be computed in theory and compared to experiment.

\subsection{Two-particle correlator}

A number of such measures can be obtained starting from a two particle correlator

$$
\left\langle\Delta n_{\boldsymbol{p}}^{\alpha} \Delta n_{\boldsymbol{k}}^{\beta}\right\rangle=\left\langle n_{\boldsymbol{p}}^{\alpha} n_{\boldsymbol{k}}^{\beta}\right\rangle-\left\langle n_{\boldsymbol{p}}^{\alpha}\right\rangle\left\langle n_{\boldsymbol{k}}^{\beta}\right\rangle
$$

where $\Delta n_{\boldsymbol{p}}^{\alpha}=n_{\boldsymbol{p}}^{\alpha}-\left\langle n_{\boldsymbol{p}}^{\alpha}\right\rangle$ is the event-by-event fluctuation of the number of particles of the type $\alpha$ in the momentum bin centered around $\boldsymbol{p}$. Experts familiar with HanburyBrown-Twiss (HBT) interferometry ${ }^{46)}$ may recognize in $(5 \cdot 2)$ the HBT correlation function.

The two-particle correlator $(5 \cdot 2)$ can be directly measured. However, for such a function of many variables, it might be difficult to represent the result of this measurement. A useful representation, for example, is obtained by limiting (projecting) the variables to transverse components of $\boldsymbol{p}$ and $\boldsymbol{k}$. The resulting plot of a function of two arguments, $p_{T}$ and $k_{T}$, is often referred to as a 'Trainor plot' (see, e.g., Ref. 47)). Interesting information can be also obtained by projecting onto the rapidities of $\boldsymbol{p}$ and $\boldsymbol{k}$. If in addition, one weights each particle with its charge, the resulting correlator, as a function of the rapidity difference $y_{\boldsymbol{p}}-y_{\boldsymbol{k}}$, is essentially the balance function introduced in. ${ }^{48)}$

There also exist many cumulative measures, proposed by theorists and/or used by experimentalists, ${ }^{42), 45), 49)-55)}$ that can be expressed in terms of correlator $(5 \cdot 2)$. As an example, the fluctuation of electric charge is given by summing over momenta $\boldsymbol{p}$ and $\boldsymbol{k}$ of all particles in the experimental acceptance window and weighting each particle with its charge $q^{\alpha}$ :

$$
\Delta Q=\sum_{\boldsymbol{p}, \alpha} q^{\alpha} \Delta n_{\boldsymbol{p}}^{\alpha} ; \quad \text { thus } \quad\left\langle(\Delta Q)^{2}\right\rangle=\sum_{\boldsymbol{p}, \alpha} \sum_{\boldsymbol{k}, \beta} q^{\alpha} q^{\beta}\left\langle\Delta n_{\boldsymbol{p}}^{\alpha} \Delta n_{\boldsymbol{k}}^{\beta}\right\rangle .
$$

The same applies to the fluctuations of the baryon charge, with $q^{\alpha}$ substituted by the baryon charge of the particles. Similar equation (see Eq.(5.5)) also applies to the fluctuations of the mean transverse momentum $p_{T}$, in which case $q^{\alpha}$ should be replaced with $p_{T}-\overline{p_{T}}$ - the deviation of the momentum $p_{T}$ from the all-event (inclusive) mean $\overline{p_{T}}$.

The correlator $(5 \cdot 2)$ can, in principle, be calculated, under assumption of thermal equilibrium, once the relevant interactions are known. In the case of the critical point, we need to concern ourselves with the interactions which can lead to singular contribution to the correlator (and, as a consequence, to susceptibilities) as the critical point is approached.

In a non-interacting gas in thermal equilibrium the correlator $(5 \cdot 2)$ vanishes unless $\boldsymbol{p}=\boldsymbol{k}$ and $\alpha=\beta .^{*}$ ) The hadrons, however, are interacting. One can ask a question: what is the effect of the interaction on the correlator $(5 \cdot 2)$ ? The answer can be found to leading order. ${ }^{56)}$ The contribution is proportional to the amplitude of

\footnotetext{
*) We are not considering HBT correlations, which are a finite size effect.
} 
the forward scattering $\mathcal{A}_{p k \rightarrow p k}$ of the particles with momenta $\boldsymbol{p}$ and $\boldsymbol{k}$. This is easy to understand using the following argument. The amplitude of the forward scattering shifts the energy of the 2-particle state relative to the sum of single particle energies. The statistical weight of the two particle state is therefore changed relative to the product of the single-particle weights. The difference is the two-particle correlator:

$$
\left\langle n_{p} n_{k}\right\rangle-\left\langle n_{p}\right\rangle\left\langle n_{k}\right\rangle=f_{p} f_{k}\left(e^{-\beta E_{I}}-1\right) \approx f_{p} f_{k}\left(-\beta E_{I}\right) \sim f_{p} f_{k} \beta \mathcal{A}_{p k \rightarrow p k} .
$$

where $f_{p}$ is the equilibrium distribution function and $E_{I}$ is the interaction energy. The exact formula, obtained using diagrammatic analysis, ${ }^{56)}$ contains additional factors $\left(1+f_{\boldsymbol{p}}\right)\left(1+f_{\boldsymbol{k}}\right)$, which can be understood as Bose enhancement (stimulated emission) factors (or, in the case of fermions, $\left(1-f_{\boldsymbol{p}}\right)\left(1-f_{\boldsymbol{k}}\right)$ - Pauli blocking).

Near the critical point the most singular contribution comes from the exchange of the sigma field quanta in the $t$ channel. $\left.{ }^{*}\right)$ Since, by kinematics, the quanta carry zero momentum, the singular contribution is proportional to $1 / m_{\sigma}^{2}$, which equals $\xi^{2}$ - the square of the sigma field correlation length.

The absolute strength of the singu-

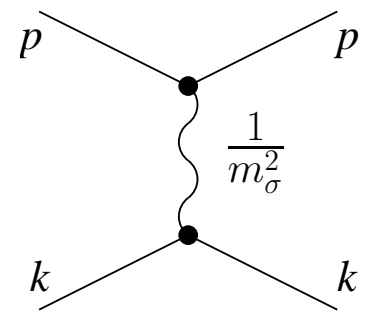

Fig. 10. Diagrammatic representation of the singular contribution to the correlator $\left\langle\Delta n_{p} \Delta n_{k}\right\rangle$. larity depends on the coupling of the critical mode sigma to the corresponding hadron in Fig. 10, which is difficult to estimate reliably. Order of magnitude estimates have been made for coupling to pions ${ }^{42)}$ and to protons ${ }^{57)}$.

As an example of the singular contribution in Fig. 10 consider baryon number susceptibility. Let $Q$ in equation $(5 \cdot 3)$ be the baryon number. Then one can see that the $1 / m_{\sigma}^{2}$, or $\xi^{2}$, singularity from Fig. 10 for scattering two baryons results in the divergence of the baryon number susceptibility $(2 \cdot 2)(\eta=0$ at this order). If only charged baryons are detected, the total baryon number cannot be measured event by event, but the number of protons is measurable. Since, according to Fig. 10, the proton number fluctuations should also be singular at the critical point, measurement of such fluctuations may provide a signal of the critical point. .57$)$

In principle, knowing the correlator $(5 \cdot 2)$ one could make quantitative predictions for fluctuation measures used in experiment. In practice, calculating the correlator is a very difficult task (what interactions should be included and what is their strength?). Non-equilibrium effects make this task even more difficult. Near the critical point these complications become less relevant since, as long as we limit ourselves to the singular effects, we only need to consider contributions such as in Fig. 10.

*) Strictly speaking, what we call here, for simplicity, "sigma" is a mixture (a linear combination) of chiral condensate, baryon density and energy density fluctuations. 


\subsection{Fluctuations, correlations, and acceptance}

Cumulative measures of fluctuations are often used to represent experimental results. These measures suffer an important drawback - they depend on the size and shape of the acceptance window of the detector. This makes comparison of different experiments, as well as an experiment to a theory, difficult. However, knowing certain properties of the correlator $(5 \cdot 2)$, it is possible to correct for acceptance in such comparisons.

As an illustration consider event-by-event fluctuations of the mean transverse momentum $p_{T}$ per particle. Most commonly used fluctuation measures are based on the width of the distribution of the event mean $p_{T}, \sigma_{\text {ebe }}^{2}$. Similar to $(5 \cdot 3), \sigma_{\text {ebe }}^{2}$ can be expressed through the correlator $(5 \cdot 2):^{42)}$

$$
\sigma_{\mathrm{ebe}}^{2}=\frac{1}{\langle N\rangle^{2}} \sum_{\boldsymbol{p} \boldsymbol{k}} \Delta p_{T} \Delta k_{T}\left\langle\Delta n_{\boldsymbol{p}} \Delta n_{\boldsymbol{k}}\right\rangle,
$$

where $\Delta p_{T} \equiv p_{T}-\overline{p_{T}}$ and $\langle N\rangle$ is the average multiplicity of accepted particles. In the thermodynamic limit $\langle N\rangle \rightarrow \infty$ the fluctuation $\sigma_{\text {ebe }}^{2}$ vanishes as $1 /\langle N\rangle$. Thus in this limit, the quantity $\langle N\rangle \sigma_{\text {ebe }}^{2}$ does not depend on the size of the system $\langle N\rangle$ and is therefore a natural subject of theoretical predictions.

To make closer comparison to experiment, it is useful to exclude the diagonal terms $\boldsymbol{p}=\boldsymbol{k}$ from the sum in $(5 \cdot 5)$, since they give the trivial statistical contribution $\langle N\rangle^{-1} \sigma_{\text {inc }}^{2}$, where $\sigma_{\text {inc }}$ is the r.m.s. width of the inclusive distribution of $p_{T}$. The remaining off-diagonal terms in (5.5) give the nontrivial "dynamical fluctuation", experimentally obtained after the subtraction:

$$
\sigma_{\text {dyn }}^{2} \equiv \sigma_{\text {ebe }}^{2}-\langle N\rangle^{-1} \sigma_{\text {inc }}^{2}
$$

In an experiment, the sum in (5.5) is limited to $\boldsymbol{p}$ and $\boldsymbol{k}$ which fall within detector acceptance. Assume, for clarity, that the acceptance is limited in rapidity, i.e., $y_{p}$ and $y_{\boldsymbol{k}}$ belong to an interval $\left[y_{\min }, y_{\max }\right]$. The cumulative measure $\sigma_{\text {ebe }}^{2}$, or $\sigma_{\text {dyn }}^{2}$, will then depend on $y_{\text {acc }} \equiv y_{\max }-y_{\min }$. This dependence simplifies in two regimes of $y_{\mathrm{acc}}$. The boundary between the two regimes is determined by the characteristic range $y_{\text {corr }}$ of the rapidity correlator of the fluctuations:

$$
\left.\langle N\rangle \sigma_{\mathrm{dyn}}^{2}\right|_{y_{\mathrm{acc}}}= \begin{cases}O\left(y_{\mathrm{acc}}\right), & \text { for } y_{\mathrm{acc}} \ll y_{\mathrm{corr}} ; \\ \left.\langle N\rangle \sigma_{\mathrm{dyn}}^{2}\right|_{\infty}, & \text { for } y_{\mathrm{acc}} \gg y_{\mathrm{corr}}\end{cases}
$$

In other words, cumulative measure $\langle N\rangle \sigma_{\text {dyn }}^{2}$ grows linearly with $y_{\text {acc }}$ for small acceptance windows and saturates at its thermodynamic limit value when the acceptance range exceeds the correlation range. In most current experiments, the width of the rapidity window $y_{\text {acc }}$ is smaller or at most comparable to the typical range of the rapidity correlator $y_{\text {corr }} \sim 1$. This means that in a typical experiment, for a cumulative measure, normalized to be finite in the thermodynamic limit, the experimentally observed magnitude is roughly proportional to the acceptance window size. ${ }^{52), 58), 59)}$ 


\subsection{Experimental results and concluding remarks}

As an example of the QCD phase diagram scan, the plot in Fig.11 shows the results of the measurements of the $p_{T}$ fluctuations using a cumulative measure $\Sigma_{p_{T}}$ described in Ref. 60). No clear non-monotonous signal, which one would expect if the experiments probed the vicinity of the critical point, was found.

It is also interesting to compare the magnitude of the observed fluctuations to the singular contribution expected near the critical point. ${ }^{42)}$ After correcting for acceptance using the method outlined in the previous section one finds:

$$
\Sigma_{p_{T}} \sim 2 \% \times\left(\frac{G}{300 \mathrm{MeV}}\right)^{2}\left(\frac{\xi}{3 \mathrm{fm}}\right)^{2},
$$

where $G$ is the magnitude of the $\sigma \pi \pi$ coupling in the diagram Fig. 10 and $\xi=1 / m_{\sigma}$.

It is important to note, that ob-

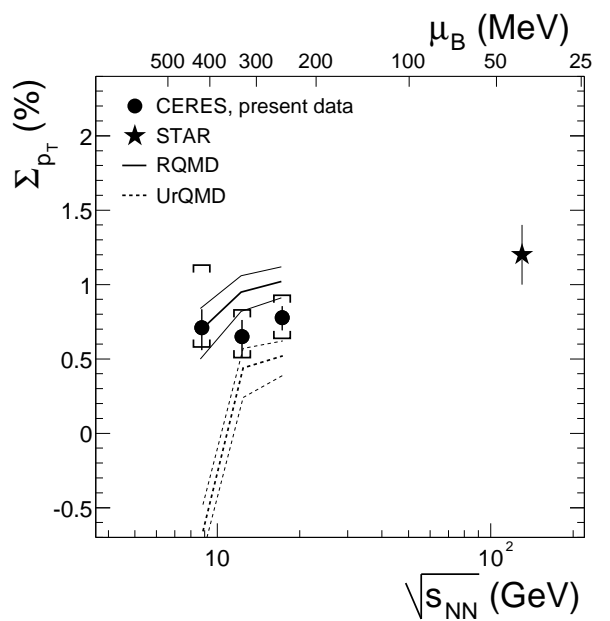

Fig. 11. CERES and STAR results for different collision energies. ${ }^{60)}$ The corresponding values of $\mu_{B}$ at freezeout are determined using statistical model analysis. ${ }^{61)}$ servation of a large magnitude of fluctuations would not by itself constitute the signal of the critical point. There are many possible contributions to the fluctuations, which are difficult to estimate. The distinct signature of the critical point is the non-monotonous behavior of fluctuation observables.

Experiments at other energies, at CERN SPS, RHIC, and future GSI facility, will be able to provide a complete scan of the reachable domain on the QCD phase diagram and either discover or rule out the presence of the critical point in this domain.

This review focused mainly on the signatures of the QCD critical point based on the event-by-event fluctuations. Further study of the properties of the critical point may reveal other, perhaps, even more sensitive and experimentally cleaner signatures. For example, real-time correlation functions and non-equilibrium dynamics near the critical point deserve further investigation. ${ }^{62)}$

Finally, the lack of a controllable and reliable theoretical method to calculate coordinates of the critical point impairs our ability to perform a more focused search. It is hard to overemphasize the importance of such a theoretical method.

\section{Acknowledgements}

The author would like to thank the organizers of the Finite density $Q C D$ workshop at Nara. He is grateful to Professor T. Kunihiro, Professor A. Nakamura and to The Yukawa Institute for Theoretical Physics for support and hospitality. 
RIKEN BNL Center and U.S. Department of Energy [DE-AC02-98CH10886] provided facilities essential for the completion of this work. This work was supported in part by DOE grant No. DE-FG0201ER41195 and by the Alfred P. Sloan Foundation.

\section{References}

1) K. Rajagopal and F. Wilczek, arXiv:hep-ph/0011333.

2) M. G. Alford, Ann. Rev. Nucl. Part. Sci. 51 (2001) 131 [arXiv:hep-ph/0102047]. arXiv:nuclth/0312007 (these proceedings).

3) T. Schafer, arXiv:hep-ph/0304281.

4) D. H. Rischke, arXiv:nucl-th/0305030.

5) D. K. Hong, Acta Phys. Polon. B 32 (2001) 1253 [arXiv:hep-ph/0101025]; arXiv:hepph/0401057 (these proceedings).

6) S. Muroya, A. Nakamura, C. Nonaka and T. Takaishi, Prog. Theor. Phys. 110 (2003) 615 [arXiv:hep-lat/0306031].

7) M. G. Alford, K. Rajagopal and F. Wilczek, Nucl. Phys. B 537 (1999) 443 [arXiv:hep$\mathrm{ph} / 9804403]$.

8) F. R. Brown et al., Phys. Rev. Lett. 65 (1990) 2491.

9) M. Asakawa and K. Yazaki, Nucl. Phys. A 504 (1989) 668.

10) A. Barducci, R. Casalbuoni, S. De Curtis, R. Gatto and G. Pettini, Phys. Lett. B 231 (1989) 463; Phys. Rev. D 41 (1990) 1610.

11) A. Barducci, R. Casalbuoni, G. Pettini and R. Gatto, Phys. Rev. D 49 (1994) 426.

12) J. Berges and K. Rajagopal, Nucl. Phys. B 538 (1999) 215 [arXiv:hep-ph/9804233].

13) M. A. Halasz, A. D. Jackson, R. E. Shrock, M. A. Stephanov and J. J. M. Verbaarschot, Phys. Rev. D 58 (1998) 096007 [arXiv:hep-ph/9804290].

14) O. Scavenius, A. Mocsy, I. N. Mishustin and D. H. Rischke, Phys. Rev. C 64 (2001), 045202 [arXiv:nucl-th/0007030].

15) N. G. Antoniou and A. S. Kapoyannis, Phys. Lett. B 563 (2003) 165 [arXiv:hepph/0211392].

16) Y. Hatta and T. Ikeda, Phys. Rev. D 67 (2003) 014028 [arXiv:hep-ph/0210284].

17) S. Gottlieb, W. Liu, D. Toussaint, R. L. Renken and R. L. Sugar, Phys. Rev. D 35 (1987) 3972; Phys. Rev. Lett. 59 (1987) 1513; Phys. Rev. D 41 (1990) 622.

18) C. W. Bernard et al., Phys. Rev. D 45 (1992) 3854.

19) M. Fukugita, H. Mino, M. Okawa and A. Ukawa, Phys. Rev. Lett. 65 (1990) 816; Phys. Rev. D 42 (1990) 2936.

20) R. D. Mawhinney, Nucl. Phys. Proc. Suppl. 30 (1993) 331.

21) D. Zhu, Nucl. Phys. Proc. Suppl. 34 (1994) 286.

22) Y. Iwasaki, K. Kanaya, S. Sakai and T. Yoshie, Z. Phys. C 71 (1996) 337 [arXiv:heplat/9504019].

23) C. W. Bernard et al., Nucl. Phys. Proc. Suppl. 42 (1995) 448 [arXiv:hep-lat/9412112].

24) See, e.g., D.J. Amit, Field theory, the renormalization group, and critical phenomena, World Scientific, 1984.

25) P. C. Hohenberg and B. I. Halperin, Rev. Mod. Phys. 49 (1977), 435.

26) R. D. Pisarski and F. Wilczek, Phys. Rev. D 29 (1984) 338.

27) K. Rajagopal and F. Wilczek, Nucl. Phys. B 399 (1993) 395 [arXiv:hep-ph/9210253].

28) K. Rajagopal, arXiv:hep-ph/9504310.

29) H. Fujii, Phys. Rev. D 67 (2003), 094018 [hep-ph/0302167]; H. Fujii and M. Ohtani, arXiv:hep-ph/0401028.

30) D. T. Son and M. A. Stephanov, arXiv:hep-ph/0401052.

31) I. M. Barbour, S. E. Morrison, E. G. Klepfish, J. B. Kogut and M. P. Lombardo, Phys. Rev. D 56 (1997) 7063 [arXiv:hep-lat/9705038].

32) Z. Fodor and S. D. Katz, JHEP 0203 (2002) 014 [arXiv:hep-lat/0106002]; arXiv:heplat/0401023 (these proceedings).

33) M. G. Alford, A. Kapustin and F. Wilczek, Phys. Rev. D 59 (1999) 054502 [arXiv:heplat/9807039].

34) F. Csikor, G.I. Egri, Z. Fodor, S.D. Katz, K.K. Szabo, A.I. Toth, hep-lat/0401022 (these 
proceedings).

35) S. Chandrasekharan, Nucl. Phys. Proc. Suppl. 94 (2001) 71 [arXiv:hep-lat/0011022].

36) U. J. Wiese, Nucl. Phys. A 702 (2002) 211.

37) J. Ambjorn, K. N. Anagnostopoulos, J. Nishimura and J. J. M. Verbaarschot, JHEP 0210 (2002) 062 [arXiv:hep-lat/0208025].

38) S. Ejiri, C. R. Allton, S. J. Hands, O. Kaczmarek, F. Karsch, E. Laermann and C. Schmidt, arXiv:hep-lat/0312006 (these proceedings).

39) P. de Forcrand and O. Philipsen, Nucl. Phys. B 673 (2003) 170; arXiv:hep-lat/0309109.

40) M. A. Stephanov, K. Rajagopal and E. V. Shuryak, Phys. Rev. Lett. 81 (1998) 4816 [arXiv:hep-ph/9806219].

41) P. Braun-Munzinger, K. Redlich and J. Stachel, arXiv:nucl-th/0304013.

$42)$ M. A. Stephanov, K. Rajagopal and E. V. Shuryak, Phys. Rev. D 60 (1999) 114028 [arXiv:hep-ph/9903292].

43) B. Berdnikov and K. Rajagopal, Phys. Rev. D 61 (2000) 105017 [arXiv:hep-ph/9912274].

44) L. Stodolsky, Phys. Rev. Lett. 75 (1995), 1044; E. V. Shuryak, Phys. Lett. B 423 (1998), 9.

45) S. A. Voloshin, V. Koch and H. G. Ritter, arXiv:nucl-th/9903060.

46) U. W. Heinz and B. V. Jacak, Ann. Rev. Nucl. Part. Sci. 49 (1999) 529 [arXiv:nuclth/9902020].

47) T. Anticic et al. [NA49 Collaboration], arXiv:hep-ex/0311009.

48) S. A. Bass, P. Danielewicz and S. Pratt, Phys. Rev. Lett. 85 (2000), 2689.

49) M. Gazdzicki and S. Mrowczynski, Z. Phys. C 54 (1992), 127; M. Gazdzicki, A. Leonidov and G. Roland, Eur. Phys. J. C 6 (1999), 365.

50) S. Mrowczynski, Phys. Lett. B 465 (1999), 8; Acta Phys. Polon. B 31 (2000), 2065.

51) S. Jeon and V. Koch, Phys. Rev. Lett. 83 (1999) 5435 [arXiv:nucl-th/9906074].

52) S. A. Voloshin [STAR Collaboration], arXiv:nucl-ex/0109006.

53) A. Bialas and V. Koch, Phys. Lett. B 456 (1999), 1; M. Belkacem, Z. Aouissat, M. Bleicher, H. Stocker and W. Greiner, arXiv:nucl-th/9903017.

54) M. Asakawa, U. W. Heinz and B. Muller, Phys. Rev. Lett. 85 (2000), 2072; S. Jeon and V. Koch, Phys. Rev. Lett. 85 (2000), 2076;

55) For a recent review of event-by-event fluctuations, see S. y. Jeon and V. Koch, arXiv:hep$\mathrm{ph} / 0304012$.

56) M. A. Stephanov, Phys. Rev. D 65 (2002) 096008 [arXiv:hep-ph/0110077].

57) Y. Hatta and M. A. Stephanov, Phys. Rev. Lett. 91 (2003) 102003. [arXiv:hep$\mathrm{ph} / 0302002]$.

58) S. y. Jeon and S. Pratt, Phys. Rev. C 65 (2002) 044902 [arXiv:hep-ph/0110043].

59) C. Pruneau, S. Gavin and S. Voloshin, Phys. Rev. C 66 (2002) 044904 [arXiv:nuclex/0204011].

60) D. Adamova et al. [CERES Collaboration], Nucl. Phys. A 727 (2003) 97 [arXiv:nuclex/0305002].

61) P. Braun-Munzinger, J. Cleymans, H. Oeschler and K. Redlich, Nucl. Phys. A 697 (2002) 902 [arXiv:hep-ph/0106066].

62) K. Fukushima, Phys. Rev. C 67 (2003) 025203 [arXiv:hep-ph/0209270]. 